Editor's Note: These short reviews of a recent paper in the Journal, written exclusively by graduate students or postdoctoral fellows, are intended to mimic the journal clubs that exist in your own departments or institutions. For more information on the format and purpose of the Journal Club, please see http://www.jneurosci.org/misc/ifa_features.shtml.

\title{
Carry on Eating: Neural Pathways Mediating Conditioned Potentiation of Feeding
}

\author{
Ben Seymour \\ Wellcome Department of Imaging Neuroscience, UCL, London WC1N 3BG, United Kingdom \\ Review of Petrovich et al. (http://www.jneurosci.org/cgi/content/full/25/36/8295)
}

Humans eat relentlessly. Our modern marketing culture, the ready availability of food, and a physiology that may well have evolved to deal with conditions of relative scarcity conspire to make us eat toward obesity. Piecing together the many different factors that influence eating behavior, from peripheral signals to motivated behavior, is of substantial interest to clinicians and, of course, food-marketing companies.

The discovery of leptin and its receptor in the early 1990s precipitated substantial research into the complex network of neuropeptide pathways that promote or inhibit feeding, focusing on what appears to be the central feeding executive, the hypothalamus. Cognitive neuroscience has also seen considerable progress in delineating the network of cortical and subcortical brain areas that solve the problem of how a hungry animal learns to find food in its often uncertain and changing environment. In a recent paper in The Journal of Neuroscience (Petrovich et al., 2005), the authors demonstrate a link between these learning paradigms and the hypothalamus.

Their study employed pavlovian appetitive conditioning, in which animals learn to predict the occurrence of food when presented with an environmental

Received 0ct. 17, 2005; accepted Dec. 30, 2005.

Correspondence should be addressed to Ben Seymour, Wellcome Department of Imaging Neuroscience, UCL, 12 Queen Square, London WC1N 3BG, UK. E-mail: bseymour@fil.ion.ucl.ac.uk.

DOI:10.1523/JNEUROSCI.4418-05.2006

Copyright $\odot 2006$ Society for Neuroscience $\quad$ 0270-6474/06/261061-002\$15.00/0 cue with which it was previously paired. The apparent simplicity of this procedure betrays a somewhat more complex set of underlying processes. According to contemporary models (Dickinson and Balleine, 2002), pavlovian learning involves direct and indirect pathways (Fig. 1): a direct pathway, by which animals learn that something of certain reward value is imminent, and an indirect pathway incorporating a more specific representation of the future reward. This latter pathway displays sensitivity to motivational state: a cue that predicts food elicits a greater response when an animal is hungry than when it is not. Importantly for obesity research, pavlovian values acquired when an animal is hungry often retain some capacity to increase feeding when the animal is subsequently sated (Weingarten, 1983). Here, the authors investigate the increase in free feeding that follows presentation of the pavlovian cue, a phenomenon termed "conditioned potentiation."

The authors show that the expression of conditioned potentiation relies on neural projections from distinct amygdalar nuclei and orbitomedial prefrontal cortex to the lateral hypothalamus, a key center for the initiation of feeding. The authors neatly combined retrograde tracing techniques to map projections to the lateral hypothalamus, with immediate-early gene expression to identify neurons functionally involved in conditioned potentiation. Rats were trained to predict the occurrence or absence of food using pavlovian auditory cues following a period of food restriction. The following day, the retrograde tracer Fluorogold was injected into the lateral hypothalamus, and animals were given ad libitum access to food until behavioral testing 2 weeks later. On the day of testing, the sated rats were again presented with each of the original pavlovian cues in sequence. As expected, the rats consumed substantially more food pellets in response to the food predicting (CS+) cue compared with the no-foodpredicting $(\mathrm{CS}-)$ cue [Petrovich et al. (2005), their Fig. $2 B$ (http://www.jneurosci. org/cgi/content/full/25/36/8295/FIG2)].

Immediately after behavioral testing, the authors identified Fluorogold-stained neurons in several key areas, including the amygdala and orbitofrontal cortex, thus identifying neurons that project to lateral hypothalamus. To identify neurons that were selectively involved in the expression of conditioned potentiation, the authors tracked mRNA concentrations of two immediate-early genes that show different temporal expression patterns: Arc, the activity of which peaks at 2-10 min after neuronal activation, and Homerla, which peaks at 25-35 min. These expression patterns coincided approximately with each of the order-randomized cue presentations during the behavioral test. Thus, by examining the relative concentrations of each mRNA, they were able to infer whether neurons were selectively activated by the CS + or CS - cue. Neurons in basolateral and basomedial amygdala, and orbitomedial prefrontal cortex were selectively activated by conditioned potentia- 
tion, and projected to lateral hypothalamus [Petrovich et al. (2005), their Fig. 4 (http://www.jneurosci.org/cgi/content/ full/25/36/8295/FIG4)]. This evidence indicates that these pathways are functionally involved in this cue-potentiated feeding during satiation.

These results provide a key functional anatomical link between rewardmotivational systems in the amygdala and prefrontal cortex with the hypothalamus, and illustrate the importance of traditional learning paradigms for obesity research. Indeed, pavlovian learning provides a fundamental building block from which a rich and diverse set of behaviors is likely to be generated (Dickinson and Balleine, 2002). Beyond conditioned potentiation, for example, recent theories of decision making rely critically on pavlovian values to guide basic action selection, a fact reflected in the success of advertising symbols in frequently persuading us to eat more than we need.

What is being encoded by the projections from amygdala and orbitomedial prefrontal cortex to the hypothalamus remains to be established. For example, do these connections convey a nonspecific reward signal that blindly increases appetitive behaviors, or is this a far more specific signal relating to specific properties of the anticipated food? One way to resolve this would be to further manipulate the motivational state, and hence specific pavlovian values, at the time of testing. For example, animals could be trained and tested in the context of specific satieties such as for fat or salt. This information would seem unlikely to project unidirectionally, because sensory specific state information, presumably computed in hypothalamus, modulates the encoding of reward value in orbitofrontal and amygdala networks. It would also be interesting to know whether these pathways

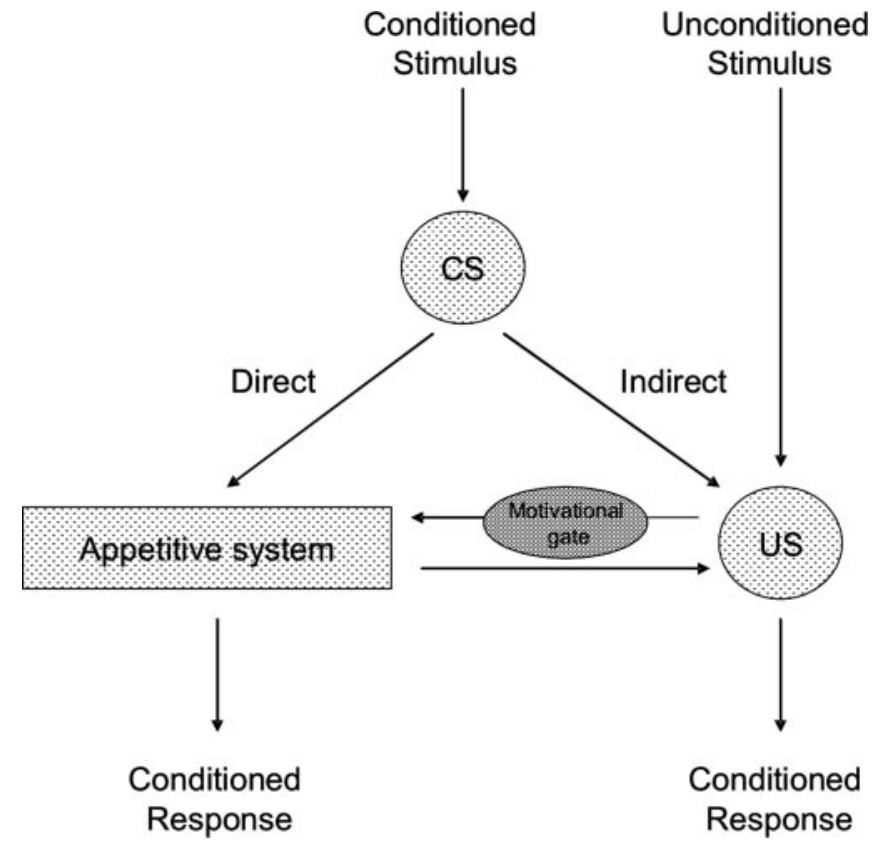

Figure 1. Konorskian model of pavlovian appetitive conditioning, showing direct and indirect pathways mediating representation of conditioned stimuli (CS) and unconditioned stimuli (US). Redrawn and adapted from Dickinson and Balleine (2002).

are involved in reinforcing the learning of instrumental behavior, particularly in the context of theoretical models of action selection (Dayan and Balleine, 2002).

Finally, the authors mention a trend toward an opposite pattern of activity in the central, compared with basolateral and basomedial, nuclei of the amygdala. If significant, this may relate to an aversive representation relating to the frustration of food omission, an idea that has substantial merit in the context of opponent models of conditioning (Solomon and Corbit, 1974). An attractive way to explore this further would be to assess projections to the hypothalamus that might be involved in conditioned suppression, by replacing the pavlovian outcome with an aversive food in the training period. Such approaches might conceivably have some therapeutic value in reversing the weight gain that afflicts so many.

\section{References}

Dayan P, Balleine BW (2002) Reward, motivation, and reinforcement learning. Neuron 36:285-298.

Dickinson A, Balleine BW (2002) The role of learning in motivation. In: Learning, motivation and emotion, Vol 3, Steven's handbook of experimental psychology, Ed 3 (Gallistel CR, ed), pp 497-533. New York: Wiley.

Petrovich GD, Holland PC, Gallagher M (2005) Amygdalar and prefrontal pathways to the lateral hypothalamus are activated by a learned cue that stimulates eating. J Neurosci 25:8295-8302.

Solomon RL, Corbit JD (1974) An opponentprocess theory of motivation. I. Temporal dynamics of affect. Psychol Rev 81:119-145.

Weingarten HP (1983) Conditioned cues elicit feeding in sated rats: a role for learning in meal initiation. Science 220:431-433. 